\title{
Pricing for a Durable-Goods Monopolist Under Rapid Sequential Innovation
}

\author{
Laura J. Kornish \\ The Fuqua School of Business, Duke University, Box 90120, Durham, North Carolina 27708-0120 \\ kornish@duke.edu
}

\begin{abstract}
durable-goods monopolist who will be introducing new and improved versions of his A product must decide how to price his products, keeping in mind the relative attractiveness of the current and future products. Dhebar (1994) has shown that if technology is changing too quickly and the producer cannot credibly commit to future prices and quality, then no equilibrium strategy exists. That is, there is no credible strategy for the future product that the producer can commit to in the first period. We show that an equilibrium pricing strategy exists if the monopolist does not offer upgrade pricing, that is, special pricing to consumers who have bought an earlier version. The author shows the possible purchase patterns in equilibrium and derives the optimal pricing strategy.

(Product Pricing; Upgrades; Technological Improvement)
\end{abstract}

\section{Introduction}

When technology is changing rapidly, consumers face a "buy or wait" decision problem in which they trade off the benefit of having the best available technology with the cost of acquiring it. Likewise, producers of sequential versions of products have a tradeoff to make. When producers introduce a sequence of new products, for example, improving versions of software, they must decide how to price the current version of the product relative to future versions of the product. In the present, the producer wants the current version to look more attractive and future versions to look less attractive. But as time passes, the once future version becomes current and the producer then wants it to look more attractive.

We extend the analysis of Dhebar (1994), who models the problem of a durable-goods monopolist selling sequential versions of a product (Versions 1 and 2) in a setting with no secondhand markets. In Dhebar's model, the producer sells only Version 1 in the first period and Version 2 in the second period. The consumers are rational; each one decides on market participation and purchase timing to maximize his surplus, benefit minus price. Dhebar looks at two cases: one in which technology is changing at a moderate pace and another in which technology is changing at a rapid pace. In the latter case, he shows that the rapidity of change leads to a situation in which there is no subgame perfect equilibrium pricing and quality strategy in which the producer earns revenue in both periods. In other words, there is no credible strategy for Version 2 that the monopolist can commit to in Period 1. In an extension with upgrades, Dhebar shows that no equilibrium exists under rapid technological change because the monopolist has the incentive to set a low second period price to attract consumers who did not buy in the first period. Dhebar $(1994,1996)$ argues that the disequilibrium imposes a demand-side constraint on how quickly improved products can be introduced.

In this paper, we use a similar set up to Dhebar (1994): The monopolist's two-period problem with rational consumers and no secondhand markets. In our model, the technological improvement is exogenous and happens between Periods 1 and 2 (in contrast to Moorthy and Png 1992, in which both products are available in the first period). We argue that an equilibrium exists for any large quality 
improvement if the monopolist does not offer any upgrade pricing, that is, special pricing for customers who have purchased previous versions. By only selling the full version of the product at a single price, the monopolist eliminates his own incentive to target the low end of the market (the people who did not buy in the first period) in the second period.

Within this context of a single price in the second period, we also ask the question that Dhebar raised: Is it really better for the producer to introduce as good a product as he can as fast as he can? Or is there a demand-side constraint which makes a moderate pace the best choice? Looking at comparative statics, we find that in some cases, the logic of profit maximization implies that a later product introduction is better. In other words, for some values of the model parameters, revenue is higher with a later introduction date for a given level of innovation in the second period. However, this is not a universal phenomenon; sometimes a faster introduction is better.

We refer the reader to the paper of Dhebar (1994) for the review of the durable-goods monopolist literature spawned by Coase (1972) and its extensions allowing for sequentially improving products. Two important developments in this area since the publication of Dhebar (1994) include Padmanabhan et al. (1997) aud Fudenberg and Tirole (1998). Padmanabhan et al. (1997) study the practice of sequential product introduction, and show that it is useful in credibly conveying a firm's private information about market potential to consumers in the presence of demand (i.e., network) externalities.

Fudenberg and Tirole (1998) analyze pricing for the sequentially innovating durable-goods monopolist under different conditions of market information: anonymous consumers with secondhand markets (e.g., textbooks), identified consumers with no secondhand markets (supercomputers), and semianonymous consumers with no secondhand markets (software). Our analysis falls in the last category. One difference between our analysis and that of Fudenberg and Tirole is that (for the last category) they concentrate on situations where the improvements in the second version are not too big (Assumption 1, p. 249), whereas rapid change is precisely the case on which we focus.
This paper contributes to the durable-goods monopolist literature by examining the case of rapidly changing technologies in a situation without secondhand markets. We derive the optimal pricing strategy in the absence of upgrade pricing offers. We introduce the model in the next section. Analysis and conclusions follow in $\S \S 3$ and 4.

\section{Model}

Our model follows Dhebar (1994). The supplier is a monopolist with zero marginal costs ${ }^{1}$ who sells sequential versions of one product. He will be introducing improved versions of the product, and he uses a strategy of product replacement: He sells only the latest generation in any period. In Period 1, only Version 1 is for sale; in Period 2, only Version 2 is for sale. All of the analytical results and examples are based on a two-period horizon for the producer.

We assume that in each period, a consumer can buy zero units or one unit of the product, consumers do not participate in secondhand markets, and consumers share discount factor $\delta$ with the producer.

The consumer's incremental valuation of generation $j$ given that he holds generation $i$ is a separable function of incremental quality $q_{i j}$ and consumer index $v$.

$$
W\left(q_{i j}, v\right)=f\left(q_{i j}\right) g(v) .
$$

Quality is assumed to be a one-dimensional metric. The increase in quality from generation $i$ to generation $j$ is denoted $q_{i j}$, and the function $f\left(q_{i j}\right)$ is increasing in $q_{i j}$. The measure $q_{i j}$ is increasing in $j$ and decreasing in $i$. The convention in this paper is that before the first period, all consumers hold nothing, or "generation $0 . "$

This separable function is similar to Dhebar (1994), with the following generalization. What we refer to as $q_{01}$ and $q_{02}$ Dhebar (1994) calls $q_{1}$ and $q_{2}$, respectively. In his analysis of upgrades, the incremental value from upgrading for consumer $v$ is $\left(f\left(q_{2}\right)-\right.$ $\left.f\left(q_{1}\right)\right) g(v)$. Our model is slightly more general in that $f\left(q_{12}\right) g(v)$, the incremental value going from Version

\footnotetext{
${ }^{1} \mathrm{Or}$, equivalently, a constant marginal cost across versions of a product.
} 
1 to Version 2 for consumer $v$, is not restricted to be $\left(f\left(q_{02}\right)-f\left(q_{01}\right)\right) g(v)$. This formulation allows for two new possibilities. First, the consumer may realize more benefit from Version 2 having used Version 1 (compared to not having used Version 1). Second, the consumer may realize less benefit from Version 2 having used Version 1. In the first case (more benefit), the consumers may have gained knowledge in using Version 1 that makes Version 2 worth more to them. In the second case (less benefit), the consumers may face some cost of "uninstalling" Version 1 so they can replace it with Version 2.

However, we do require that

$$
f\left(q_{01}\right)+\delta f\left(q_{12}\right)>\delta f\left(q_{02}\right),
$$

which implies that if everything were free, it is better to have Version 1 and then switch to Version 2 than it is to wait for Version 2. This assumption places a limit on the size of the switching cost. The size of the learning effect is limited by the assumption that $q_{i j}$ is decreasing in $i$, namely $f\left(q_{02}\right)>f\left(q_{12}\right)$.

The index $v$ ranges from 0 to 1 , with 0 representing the lowest-end consumer and 1 representing the highest-end consumer. The consumers are distributed uniformly between 0 and 1 . The function $g(v)$ is assumed to be nonnegative, strictly increasing, and normalized: $g(0)=0, g(1)=1$, and $g^{\prime}(v)>0$. Further, we impose the condition that $(1-v) g(v)$ is concave to ensure that the single-period profit maximizing problem has a unique solution. We represent the inverse of $g()$ as

$$
h(\cdot) \equiv g^{-1}(\cdot) .
$$

We define the single-period profit maximizing price $p_{01}^{*}$ for the first period

$$
p_{01}^{*}=\arg \max _{p_{01}} p_{01}\left(1-h\left(\frac{p_{01}}{f\left(q_{01}\right)}\right)\right) .
$$

Finally, we restrict our attention to the case of rapid change, with technology improving in present value, i.e.,

$$
f\left(q_{01}\right)<\delta f\left(q_{02}\right)
$$

This is the case for which Dhebar (1994) shows that an equilibrium cannot exist under the upgrade pricing scheme.

\section{Analysis}

In this section, we look at the existence and nature of equilibrium solutions to the two-period problem in which the producer does not offer special upgrade pricing to people who bought in the first period. In $\S 3.1$, we derive the consumer purchase patterns for any given set of prices $\left(p_{1}, p_{2}\right)$. In $\$ 3.2$, we use the derivation of consumer behavior to analyze producer strategy.

\subsection{Consumer Purchase Patterns}

Each consumer $v \in[0,1]$ weighs the four alternatives: (1) Don't buy in either period, with value $0,(2)$ buy in the first period only, with value $f\left(q_{01}\right) g(v)-p_{1}$, (3) buy in the second period only, with value $\delta\left(f\left(q_{02}\right) g(v)-\right.$ $\left.p_{2}\right)$, and (4) buy in the first period and in the second period, with value $f\left(q_{01}\right) g(v)-p_{1}+\delta\left(f\left(q_{12}\right) g(v)-\right.$ $\left.p_{2}\right)$. Comparing each alternative with the others, we describe the parts of the $v$ continuum in which each of the four strategies is optimal.

1. The strategy of not buying in either period is optimal for

$$
\begin{aligned}
\{v: 0 & \leq v \leq 1, g(v) \\
& \left.<\min \left(\frac{p_{1}}{f\left(q_{01}\right)}, \frac{p_{2}}{f\left(q_{02}\right)}, \frac{p_{1}+\delta p_{2}}{f\left(q_{01}\right)+\delta f\left(q_{12}\right)}\right)\right\} .
\end{aligned}
$$

2. The strategy of buying in the first period only is optimal for

$$
\begin{aligned}
\{v: 0 & \leq v \leq 1, \frac{p_{1}}{f\left(q_{01}\right)} \leq g(v) \\
& \left.<\min \left(\frac{\delta p_{2}-p_{1}}{\delta f\left(q_{02}\right)-f\left(q_{01}\right)}, \frac{p_{2}}{f\left(q_{12}\right)}\right)\right\} .
\end{aligned}
$$

3. The strategy of buying in the second period only is optimal for

$$
\begin{aligned}
\{v: 0 & \leq v \leq 1, \max \left(\frac{p_{2}}{f\left(q_{02}\right)}, \frac{\delta p_{2}-p_{1}}{\delta f\left(q_{02}\right)-f\left(q_{01}\right)}\right) \\
& \left.\leq g(v)<\frac{p_{1}}{f\left(q_{01}\right)+\delta f\left(q_{12}\right)-\delta f\left(q_{02}\right)}\right\} .
\end{aligned}
$$



for

4. The strategy of buying in both periods is optimal

$$
\begin{gathered}
\left\{v: 0 \leq v \leq 1, g(v) \geq \max \left(\frac{p_{1}+\delta p_{2}}{f\left(q_{01}\right)+\delta f\left(q_{12}\right)}, \frac{p_{2}}{f\left(q_{12}\right)},\right.\right. \\
\left.\left.\frac{p_{1}}{f\left(q_{01}\right)+\delta f\left(q_{12}\right)-\delta f\left(q_{02}\right)}\right)\right\} .
\end{gathered}
$$

The four parts must form a mutually exclusive and collectively exhaustive partition of the $v$ (or $g(v)$ ) line. Below we explain the three possible partitions or purchase patterns corresponding to three regions in $\left(p_{1}, p_{2}\right)$ space (see Figures 1 and 2).

Proposition 1. For any combination of nonnegative $\left(p_{1}, p_{2}\right)$, one of three cases holds. (Note: The additional condition $0 \leq v \leq 1$ is part of each of the set definitions below.)

REGION I. If

$$
p_{2} \geq \frac{f\left(q_{12}\right) p_{1}}{f\left(q_{01}\right)+\delta f\left(q_{12}\right)-\delta f\left(q_{02}\right)},
$$

then the purchase pattern has three parts. The $\{v: g(v)<$ $\left.p_{1} / f\left(q_{01}\right)\right\}$ buy nothing; the $\left\{v: p_{1} / f\left(q_{01}\right) \leq g(v)<\right.$ $\left.p_{2} / f\left(q_{12}\right)\right\}$ buy in the first period only; the $\{v: g(v) \geq$ $\left.p_{2} / f\left(q_{12}\right)\right\}$ buy in both periods.

REGION II. If

$$
\frac{f\left(q_{02}\right) p_{1}}{f\left(q_{01}\right)} \leq p_{2} \leq \frac{f\left(q_{12}\right) p_{1}}{f\left(q_{01}\right)+\delta f\left(q_{12}\right)-\delta f\left(q_{02}\right)},
$$

Figure 1 The Three Regions in $\left(p_{1}, p_{2}\right)$ Space Corresponding to the Three Possible Consumer Purchase Patterns

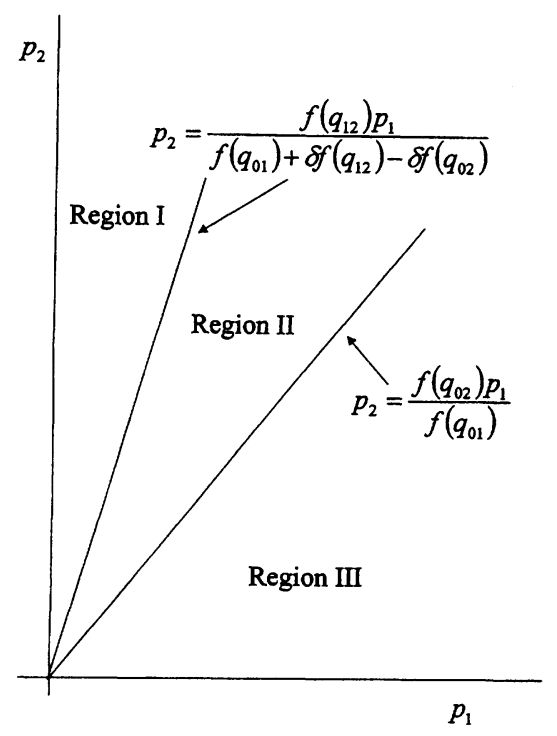

Figure 2 The Purchase Patterns in the Three Regions

Region I

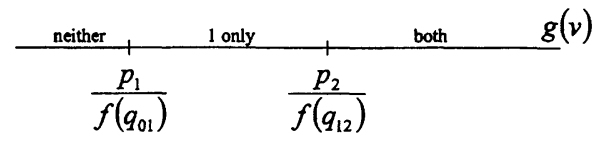

Region II

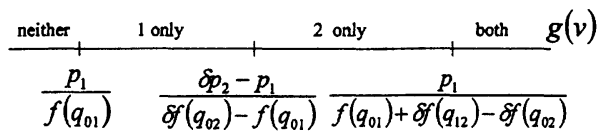

Region III

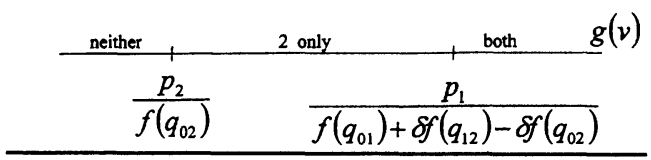

then the purchase pattern has four parts. The $\{v: g(v)<$ $\left.p_{1} / f\left(q_{01}\right)\right\}$ buy nothing; the

$$
\left\{v: \frac{p_{1}}{f\left(q_{01}\right)} \leq g(v)<\frac{\delta p_{2}-p_{1}}{\delta f\left(q_{02}\right)-f\left(q_{01}\right)}\right\}
$$

buy in the first period only; the

$$
\left\{v: \frac{\delta p_{2}-p_{1}}{\delta f\left(q_{02}\right)-f\left(q_{01}\right)} \leq g(v)<\frac{p_{1}}{f\left(q_{01}\right)+\delta f\left(q_{12}\right)-\delta f\left(q_{02}\right)}\right\}
$$

buy in the second period only; the $\left\{v: g(v) \geq p_{1} /\left(f\left(q_{01}\right)+\right.\right.$ $\left.\left.\delta f\left(q_{12}\right)-\delta f\left(q_{02}\right)\right)\right\}$ buy in both periods.

REGION III. If $p_{2} \leq f\left(q_{02}\right) p_{1} / f\left(q_{01}\right)$, then the purchase pattern has three parts. The $\left\{v: g(v)<p_{2} / f\left(q_{02}\right)\right\}$ buy nothing; the $\left\{v: p_{2} / f\left(q_{02}\right) \leq g(v)<p_{1} /\left(f\left(q_{01}\right)+\delta f\left(q_{12}\right)-\right.\right.$ $\left.\left.\delta f\left(q_{02}\right)\right)\right\}$ buy in the second period only; the $\{v: g(v) \geq$ $\left.p_{1} /\left(f\left(q_{01}\right)+\delta f\left(q_{12}\right)-\delta f\left(q_{02}\right)\right)\right\}$ buy in both periods.

Proof. See the appendix.

These three cases cover the entire $\left(p_{1}, p_{2}\right)$ space as shown in Figure 1. In all three possibilities (see Figure 2), higher-end consumers are buying more often and/or higher versions than lower-end consumers. The three regions are characterized by which version of the product is priced more aggressively relative to the other. In Region I, it is Version 1; in Region II, it is neither version; in Region III, it is Version 2.

Region I can be characterized as the "low introductory price" region; there is a limit to how high 
$p_{1}$ can be relative to $p_{2}$ in this region. Because the price of Version 1 is comparatively low, that version is attractive to a large segment of consumers. Some of those consumers (the ones at the high end) also buy Version 2.

In Region II, there is both a limit to how high $p_{1}$ can be relative to $p_{2}$ and a limit to how high $p_{2}$ can be relative to $p_{1}$. This is the "moderation" region-neither product is priced in an aggressive manner relative to the other. In Region II, we see what Fudenberg and Tirole (1998) call leapfrogging: Some people who bought in the first period don't buy in Period 2 and some who didn't buy in Period 1 do buy in Period 2.

Finally, in Region III, there is a limit to how high $p_{2}$ can be relative to $p_{1}$. This scenario features a very desirable Version 2; not only is the product greatly improved, but the price is attractive relative to Version 1. Consequently, the purchase pattern in this region includes people who wait for Version 2 and does not include any people who only buy Version 1 .

The next step in this analysis is to determine which of the three purchase patterns are consistent with optimizing behavior on the part of the producer.

\subsection{Producer Optimization}

Each of the three regions from Proposition 1 presents a constrained optimization problem. The objective function (revenue) is dictated by the purchase pattern associated with the region, and the constraints come from two sources. The first source of constraints is the condition for which the purchase pattern holds (for example, $p_{2} \leq f\left(q_{02}\right) p_{1} / f\left(q_{01}\right)$, for the third case), and the second source of constraints is the condition that the producer sell in both periods.

3.2.1. Region I. According to the purchase pattern for the first region, the highest valuation consumers buy in both periods, the middle valuation consumers buy in the first period only, and the lowest valuation consumers do not buy at all.

To ensure subgame perfection, we solve the secondperiod problem first. In the second period, the producer sells to $\left\{v: g(v) \geq p_{2} / f\left(q_{12}\right)\right\}$, and so he solves the following problem:

$$
R_{2}^{I^{*}}\left(p_{1}, p_{2}\right)=\max _{p_{2}} p_{2}\left(1-h\left(\frac{p_{2}}{f\left(q_{12}\right)}\right)\right)
$$

s.t.

$$
\begin{aligned}
& p_{2} \geq 0 \\
& p_{2} \leq f\left(q_{12}\right) \\
& p_{2} \geq \frac{f\left(q_{12}\right) p_{1}}{f\left(q_{01}\right)+\delta f\left(q_{12}\right)-\delta f\left(q_{02}\right)} .
\end{aligned}
$$

The objective function is second-period revenue. Constraints (11) and (12) ensure price and quantity nonnegativity, respectively. Constraint (13) is the inequality defining Region I. This problem is feasible if and only if $p_{1} \leq f\left(q_{01}\right)+\delta f\left(q_{12}\right)-\delta f\left(q_{02}\right)$, so that the lower bound on $p_{2}$ from (13) is lower than the upper bound on $p_{2}$ from (12).

In the first period, the producer sells to $\{v: g(v) \geq$ $\left.p_{1} / f\left(q_{01}\right)\right\}$, and so he solves the following problem:

$$
\begin{aligned}
R^{I^{*}}\left(p_{1}, p_{2}\left(p_{1}\right)\right)= & \max _{p_{1}} p_{1}\left(1-h\left(\frac{p_{1}}{f\left(q_{01}\right)}\right)\right) \\
& +\delta R_{2}^{I^{*}}\left(p_{1}, p_{2}\left(p_{1}\right)\right)
\end{aligned}
$$

s.t.

$$
\begin{aligned}
& p_{1} \geq 0 \\
& p_{1} \leq f\left(q_{01}\right)+\delta f\left(q_{12}\right)-\delta f\left(q_{02}\right) .
\end{aligned}
$$

Proposition 2. Under Conditions (2) and (5),

$$
\begin{aligned}
& p_{1}^{I^{*}}=\frac{\left(f\left(q_{01}\right)+\delta f\left(q_{12}\right)-\delta f\left(q_{02}\right)\right) p_{01}^{*}}{f\left(q_{01}\right)} \text { and } \\
& p_{2}^{I^{*}}=\frac{f\left(q_{12}\right) p_{01}^{*}}{f\left(q_{01}\right)}
\end{aligned}
$$

constitute a subgame perfect equilibrium pricing strategy with sales in both periods.

Proof. See the appendix.

If the producer charges any $p_{1}$ between 0 and $\left(f\left(q_{01}\right)+\delta f\left(q_{12}\right)-\delta f\left(q_{02}\right)\right) p_{01}^{*} / f\left(q_{01}\right)$ and the consumers expect a $p_{2}=f\left(q_{12}\right) p_{01}^{*} / f\left(q_{01}\right)$, then it makes sense for the producer to charge that $p_{2}$. Profits are maximized for the producer by charging the highest $p_{1}$ in that range. Further, there cannot be a subgame perfect equilibrium under the Region I purchase pattern if $p_{1} \leq\left(f\left(q_{01}\right)+\delta f\left(q_{12}\right)-\delta f\left(q_{02}\right)\right) p_{01}^{*} / f\left(q_{01}\right)$ does not hold. Setting $p_{1}$ higher than $\left(f\left(q_{01}\right)+\delta f\left(q_{12}\right)-\right.$ $\left.\delta f\left(q_{02}\right)\right) p_{01}^{*} / f\left(q_{01}\right)$ implies that the Region I purchase pattern constraint (13) is binding. In other words, the 
producer's second-period price would have to be governed by a concern for eliminating consumer regret rather than profit maximization.

In the equilibrium given in Proposition 2, the firstperiod price is lower than $p_{01}^{*}$, the single-period profit maximizing price. This purchase pattern-low valuers buy nothing, middle valuers buy 1 only, high valuers buy both-is based on selling to a "large" group of consumers in the first period and selling to some of them again in the second period. To get the "large" base in the first period, the first-period price is low.

Proposition 2 shows that the "low introductory price" strategy is feasible in the sense that there is a Version 2 price that the producer can promise to charge that will not cause any consumers to regret their decisions. Conceptually, a low introductory price is appealing because it makes Version 1 attractive based on price; Version 2 will be a big performance improvement over Version 1, so a low price for Version 1 encourages first-period sales.

3.2.2. Region II. The second region has a purchase pattern with four parts (from lowest to highest valuation): Buy nothing, buy Version 1 only, buy Version 2 only, and buy both. In the second-period problem of the second region, the producer can only change price, not quantity. The second-period quantity is determined in the first period by the consumer indifferent between Version 1 only and Version 2 only. For what follows, we define $\hat{v}=\min (1$, $h\left(\left(\delta p_{2}-p_{1}\right) /\left(\delta f\left(q_{02}\right)-f\left(q_{01}\right)\right)\right)$.

In the second period, the producer sells to $\{v: v \geq$ $\hat{v}\}$, and so he solves the following problem:

$$
R_{2}^{I I^{*}}\left(p_{1}, p_{2}\right)=\max _{p_{2}} p_{2}(1-\hat{v})
$$

s.t.

$$
\begin{aligned}
& p_{2} \geq 0 \\
& p_{2} \geq \frac{p_{1} f\left(q_{02}\right)}{f\left(q_{01}\right)} \\
& p_{2} \leq \frac{p_{1} f\left(q_{12}\right)}{f\left(q_{01}\right)+\delta f\left(q_{12}\right)-\delta f\left(q_{02}\right)} .
\end{aligned}
$$

Constraints (21) and (22) are the conditions associated with the purchase pattern in Region II.

The solution to this problem is to make $p_{2}$ as big as possible. That is, set $p_{2}$ to its upper bound, $p_{2}=p_{1} f\left(q_{12}\right) /\left(f\left(q_{01}\right)+\delta f\left(q_{12}\right)-\delta f\left(q_{02}\right)\right)$. When we set $p_{2}$ in such a way, the purchase pattern collapses from four into three parts. The $\left\{v: g(v)<p_{1} / f\left(q_{01}\right)\right\}$ buy nothing; the

$\left\{v: \frac{p_{1}}{f\left(q_{01}\right)} \leq g(v)<\frac{p_{1}}{f\left(q_{01}\right)+\delta f\left(q_{12}\right)-\delta f\left(q_{02}\right)}=\frac{p_{2}}{f\left(q_{12}\right)}\right\}$

buy in the first period only; the

$$
\left\{v: g(v) \geq \frac{p_{1}}{f\left(q_{01}\right)+\delta f\left(q_{12}\right)-\delta f\left(q_{02}\right)}=\frac{p_{2}}{f\left(q_{12}\right)}\right\}
$$

buy in both periods. This purchase pattern is the same as the purchase pattern for Region I. Because the equilibrium pricing result in Proposition 2 is on the boundary between the two regions, that equilibrium pricing scheme applies to this second case as well

The conclusion we draw from this border solution is that there does not exist a feasible (i.e., profit maximizing without regretful consumers) pricing scheme that promotes leapfrogging. When technology is changing rapidly, and consumers will have a chance to buy Version 2, pricing Version 2 unaggressively results in the "low introductory price" scenario in which all consumers above a threshold buy Version 1 and then the high end of that group also buys Version 2. This result highlights the difficulty in making Version 1 relatively more attractive to some consumers while simultaneously making Version 2 relatively more attractive to others.

3.2.3. Region III. In the purchase pattern for the third region, the lowest valuation consumers buy nothing, the middle valuation consumers buy in the second period only, and the highest valuation consumers buy in both periods.

In the second period, the producer sells to $\left\{v: g(v) \geq p_{2} / f\left(q_{02}\right)\right\}$, and so he solves the following problem:

$$
R_{2}^{I I I^{*}}\left(p_{1}, p_{2}\right)=\max _{p_{2}} p_{2}\left(1-h\left(\frac{p_{2}}{f\left(q_{02}\right)}\right)\right)
$$

s.t.

$$
\begin{aligned}
& p_{2} \geq 0 \\
& p_{2} \leq f\left(q_{02}\right) \\
& p_{2} \leq \frac{f\left(q_{02}\right) p_{1}}{f\left(q_{01}\right)} .
\end{aligned}
$$


This problem is feasible if and only if $p_{1} \geq 0$ (so that the lower bound is lower than the upper bound on $p_{2}$ ).

In the first period, the producer sells to

$$
\left\{v: g(v) \geq \frac{p_{1}}{f\left(q_{01}\right)+\delta f\left(q_{12}\right)-\delta f\left(q_{02}\right)}\right\},
$$

and so he solves

$$
\begin{aligned}
R^{I I I^{*}}= & \max _{p_{1}} p_{1}\left(1-h\left(\frac{p_{1}}{f\left(q_{01}\right)+\delta f\left(q_{12}\right)-\delta f\left(q_{02}\right)}\right)\right) \\
& +\delta R_{2}^{I I I^{*}}\left(p_{1}, p_{2}\left(p_{1}\right)\right)
\end{aligned}
$$

s.t.

$$
\begin{aligned}
& p_{1} \geq 0 \\
& p_{1} \leq f\left(q_{01}\right)+\delta f\left(q_{12}\right)-\delta f\left(q_{02}\right) .
\end{aligned}
$$

Constraints (28) and (29) ensure price and quantity nonnegativity, respectively.

Proposition 3. Under Conditions (2) and (5), and if

$$
p_{01}^{*}<f\left(q_{01}\right)+\delta f\left(q_{12}\right)-\delta f\left(q_{02}\right),
$$

then

$$
\begin{aligned}
& p_{1}^{I I I^{*}}=p_{01}^{*} \quad \text { and } \\
& p_{2}^{I I I^{*}}=\frac{f\left(q_{02}\right) p_{01}^{*}}{f\left(q_{01}\right)}
\end{aligned}
$$

constitute a subgame perfect equilibrium pricing strategy with sales in both periods.

Proof. See the appendix.

If the producer charges any $p_{1}$ between $p_{01}^{*}$ and $f\left(q_{01}\right)+\delta f\left(q_{12}\right)-\delta f\left(q_{02}\right)$ and the consumers expect a $p_{2}=f\left(q_{02}\right) p_{01}^{*} / f\left(q_{01}\right)$, then it makes sense for the producer to charge that $p_{2}$. Profits are maximized for the producer by charging the lowest $p_{1}$ in that range. Further, there cannot be a subgame perfect equilibrium under the Region III purchase pattern if $p_{1} \geq p_{01}^{*}$ does not hold. Setting $p_{1}$ lower than $p_{01}^{*}$ implies that the Region III purchase pattern constraint (26) is binding. In other words, the producer's second-period price would have to be governed by a concern for eliminating consumer regret rather than profit maximization.

Proposition 3 shows that for some shapes of $g(v)$ and some values of the parameters, the strategy of
Figure 3 Equilibrium Points: Point A (Region I) and Point B (Region III). This Figure Is Drawn such that $p_{01}^{*}<f\left(q_{01}\right)+\delta f\left(q_{12}\right)-$ $\delta f\left(q_{02}\right)$

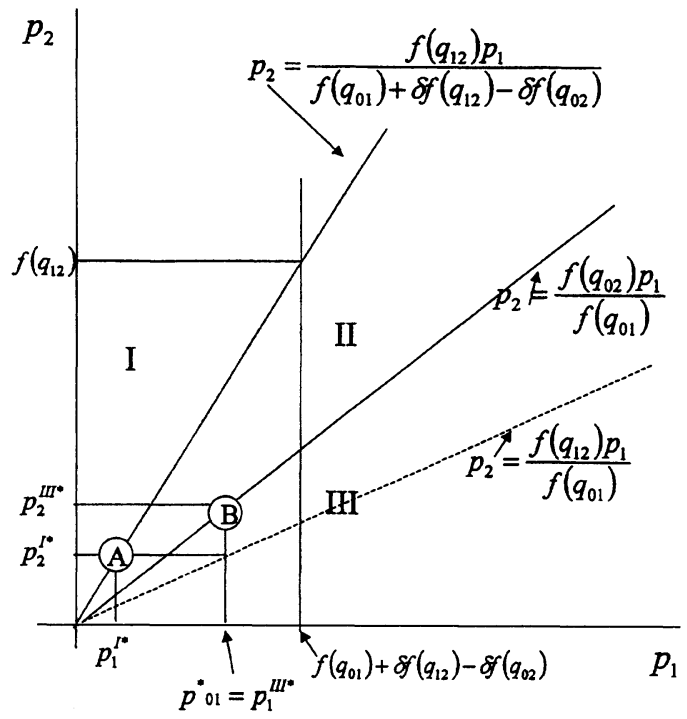

playing up Version 2 can work. Because Version 2 is such a big improvement over Version 1 and it is being priced aggressively, only the highest-end consumers will purchase Version 1 , and then all of them will also buy Version 2. Region III is the "Super Version 2" region: Version 2 is greatly improved and relatively well priced compared to Version 1 . Looking at Figure 3, we can see that the prices for both versions under this pricing strategy are higher than the respective prices under the Region I pricing strategy given in Proposition 2. In Region III, there is a limit to how high $p_{2}$ can be for a given $p_{1}$. By offering a good price for the advanced product, prices for both versions are higher compared to Region I in which a low price for Version 1 drives the strategy. In the next section, we address the relative performance of the strategies suggested by Propositions 2 and 3 .

3.2.4. Summary of the Regions. The two possible equilibria are shown in Figure 3. The analysis of Region I tells us that under Assumptions (2) and (5), there exists a subgame perfect equilibrium pricing strategy with sales in both periods given by Equations (17) and (18). The solution has a low first-period price with a large number of consumers purchasing. Everyone who values Version 1 more than $p_{1}$ buys in the first period. The first-period price is low relative to 
the single-period profit maximizing price. This strategy is based on pricing Version 1 aggressively and selling to a large group in the first period, and then selling again to a group of the highest valuers in the second period.

From the analysis of Region III, we see that under Assumptions (2) and (5) if the additional condition (30) is met, then there is also a subgame perfect equilibrium pricing strategy with sales in both periods given by (31) and (32). In this case, the first-period price is the single-period optimizing price, but not all people who value at that price buy; only people who are at the very high end purchase in the first period. In the second period, all first-period purchasers buy again, as well as some people who did not buy in the first period.

The question then arises about which of these equilibria should the producer choose. The expression for revenue for the point in Proposition 2 is given by

$$
\begin{aligned}
R^{I^{*}}= & p_{01}^{*} \frac{f\left(q_{01}\right)+\delta f\left(q_{12}\right)-\delta f\left(q_{02}\right)}{f\left(q_{01}\right)} \\
& \times\left(1-h\left(\frac{\left(f\left(q_{01}\right)+\delta f\left(q_{12}\right)-\delta f\left(q_{02}\right)\right) p_{01}^{*}}{f\left(q_{01}\right)^{2}}\right)\right) \\
& +\delta p_{01}^{*} \frac{f\left(q_{12}\right)}{f\left(q_{01}\right)}\left(1-h\left(\frac{p_{01}^{*}}{f\left(q_{01}\right)}\right)\right) .
\end{aligned}
$$

The expression for revenue for the point in Proposition 3 is given by

$$
\begin{aligned}
R^{I I I^{*}}= & p_{01}^{*}\left(1-h\left(\frac{p_{01}^{*}}{f\left(q_{01}\right)+\delta f\left(q_{12}\right)-\delta f\left(q_{02}\right)}\right)\right) \\
& +\delta p_{01}^{*} \frac{f\left(q_{02}\right)}{f\left(q_{01}\right)}\left(1-h\left(\frac{p_{01}^{*}}{f\left(q_{01}\right)}\right)\right) .
\end{aligned}
$$

If Condition (30) does not hold, there is no reason for comparison. If Condition (30) does hold, then the equilibrium in Region I yields higher revenues than the equilibrium in Region III if and only if

$$
\begin{aligned}
& \frac{f\left(q_{01}\right)+\delta f\left(q_{12}\right)-\delta f\left(q_{02}\right)}{f\left(q_{01}\right)} \\
& \quad \times\left(1-h\left(\frac{\left(f\left(q_{01}\right)+\delta f\left(q_{12}\right)-\delta f\left(q_{02}\right)\right) p_{01}^{*}}{f\left(q_{01}\right)^{2}}\right)\right) \\
& -\left(1-h\left(\frac{p_{01}^{*}}{f\left(q_{01}\right)+\delta f\left(q_{12}\right)-\delta f\left(q_{02}\right)}\right)\right) \\
& >\frac{\delta\left(f\left(q_{02}\right)-f\left(q_{12}\right)\right)}{f\left(q_{01}\right)}\left(1-h\left(\frac{p_{01}^{*}}{f\left(q_{01}\right)}\right)\right) .
\end{aligned}
$$

The LHS of (35) represents the increase in first-period revenue from Region I pricing over Region III pricing. The RHS represents the increase in second-period revenue from Region III pricing over Region I pricing.

\subsection{Timing Considerations}

With the expressions for revenue, we can address the issue of timing the introduction of the second product. The period length is exogenous in our model; we are not playing a game of introduction timing. We address the timing issue by looking at the comparative statics, how revenue changes with a change in the discount factor $\delta$. On the one hand, if the revenue function is increasing in $\delta$, then the monopolist's revenue is increased by introducing the product sooner. However, because of technical constraints, the product may not be available sooner. On the other hand, if the revenue function is decreasing in $\delta$, then the monopolist's revenue is increased by introducing the product later. This benefit from introducing the product later is similar to the issue that Dhebar (1994) raises, a demand-side constraint.

We show that there exist regions of the parameter space for which the revenue function is increasing in $\delta$ and regions for which the revenue function is decreasing in $\delta$. Let $g(v)=v\left(\Rightarrow p_{01}^{*}=f\left(q_{01}\right) / 2\right), f\left(q_{01}\right)=1$, $f\left(q_{12}\right)=1.5$, and $f\left(q_{02}\right)=2.5$. To satisfy (5), we require that $\delta>0.4$. The revenue for the Region I equilibrium point is

$$
R^{I}=\frac{(1-\delta)(1+\delta)}{4}+\frac{1.5 \delta}{4}
$$

The condition for an equilibrium point to exist in Region III (30) translates to $\delta>0.5$. The revenue for the Region III equilibrium point is

$$
R^{I I I}=\frac{1}{4} \frac{1-2 \delta}{1-\delta}+\frac{2.5 \delta}{4} .
$$

We find which equilibrium point yields higher revenues given a value of $\delta$. For all $\delta>0.5$, the $R^{I}$ $>R^{I I I}$.

The expression for $R^{I}$ is increasing in $\delta$ for $\delta<0.75$ and decreasing for $\delta>0.75$. For $0.4<\delta<0.75$, faster is better: Revenue would be higher if the period were shorter. But for $0.75<\delta<1$, the demand-side issue that Dhebar (1994) raises takes effect: Delay increases revenue. 


\section{Conclusion}

In this paper, we have extended the analysis of Dhebar (1994), probing his lack of equilibrium conclusion in a game in which a monopolist selling sequential versions faces rational consumers. We find that if the monopolist does not segment the market in the second period, that is, if he does not offer special upgrade pricing, then an equilibrium pricing strategy exists even when technology is improving in the present value terms.

One issue we must address is the credibility of the single second-period price strategy. To answer that, we must look outside the model itself for a context. If the producer makes it either too easy for a consumer to claim he bought in the first period or too difficult to prove he bought in the first period, then he could credibly charge one price in the second period.

Given that we do find equilibrium prices, we examine Dhebar's (1994) assertion that faster introduction is not better for the producer when technology is improving in present value terms. Comparative statics reveal that sometimes faster introduction is better. To extend the analysis, we could examine a game with the timing of the second period as a decision variable for the producer.

\section{Acknowledgments}

The author gratefully acknowledges the assistance of Sam Chiu, John Weyant, and Blake Johnson from the Department of Engineering-Economic Systems and Operations Research at Stanford University and the helpful comments of two anonymous reviewers. This work was supported by both the National Science Foundation Graduate Fellowship and the Gerald Lieberman Fellowship from Stanford.

\section{Appendix}

Proof of Proposition 1.

Region I,

$$
p_{2} \geq \frac{f\left(q_{12}\right) p_{1}}{f\left(q_{01}\right)+\delta f\left(q_{12}\right)-\delta f\left(q_{02}\right)} .
$$

Find the upper bound of the buy-nothing region:

$$
\begin{aligned}
\left(\frac{f\left(q_{02}\right)}{f\left(q_{01}\right)}\right. & \leq \frac{f\left(q_{12}\right)}{f\left(q_{01}\right)+\delta f\left(q_{12}\right)-\delta f\left(q_{02}\right)} \text { and } \\
p_{2} & \left.\geq \frac{f\left(q_{12}\right) p_{1}}{f\left(q_{01}\right)+\delta f\left(q_{12}\right)-\delta f\left(q_{02}\right)}\right) \Rightarrow \frac{p_{1}}{f\left(q_{01}\right)} \leq \frac{p_{2}}{f\left(q_{02}\right)}, \quad \text { and } \\
\frac{p_{1}}{f\left(q_{01}\right)} & \leq \frac{p_{2}}{f\left(q_{02}\right)} \Rightarrow \frac{p_{1}}{f\left(q_{01}\right)} \leq \frac{p_{2}}{f\left(q_{12}\right)} \Leftrightarrow \frac{p_{1}}{f\left(q_{01}\right)} \leq \frac{p_{1}+\delta p_{2}}{f\left(q_{01}\right)+\delta f\left(q_{12}\right)} .
\end{aligned}
$$

So $v: g(v)=p_{1} / f\left(q_{01}\right)$ is the upper bound.

Find the upper bound of the buy in the first-period-only region:

$$
p_{2} \geq \frac{f\left(q_{12}\right) p_{1}}{f\left(q_{01}\right)+\delta f\left(q_{12}\right)-\delta f\left(q_{02}\right)} \Leftrightarrow \frac{p_{2}}{f\left(q_{12}\right)} \leq \frac{\delta p_{2}-p_{1}}{\delta f\left(q_{02}\right)-f\left(q_{01}\right)} .
$$

So $v: g(v)=p_{2} / f\left(q_{12}\right)$ is the upper bound.

No consumers buy in the second period only. The upper bound of the second-period-only region is below the lower bound:

$$
\begin{aligned}
p_{2} & \geq \frac{f\left(q_{12}\right) p_{1}}{f\left(q_{01}\right)+\delta f\left(q_{12}\right)-\delta f\left(q_{02}\right)} \Leftrightarrow \frac{p_{1}}{f\left(q_{01}\right)+\delta f\left(q_{12}\right)-\delta f\left(q_{02}\right)} \\
& \leq \frac{\delta p_{2}-p_{1}}{\delta f\left(q_{02}\right)-f\left(q_{01}\right)} .
\end{aligned}
$$

Find the lower bound of the buy in both periods region:

$$
\begin{aligned}
p_{2} & \geq \frac{f\left(q_{12}\right) p_{1}}{f\left(q_{01}\right)+\delta f\left(q_{12}\right)-\delta f\left(q_{02}\right)} \Rightarrow p_{2} \geq \frac{f\left(q_{12}\right) p_{1}}{f\left(q_{01}\right)} \\
& \Leftrightarrow \frac{p_{2}}{f\left(q_{12}\right)} \geq \frac{p_{1}+\delta p_{2}}{f\left(q_{01}\right)+\delta f\left(q_{12}\right)}, \quad \text { and } \\
p_{2} & \geq \frac{f\left(q_{12}\right) p_{1}}{f\left(q_{01}\right)+\delta f\left(q_{12}\right)-\delta f\left(q_{02}\right)} \\
& \Leftrightarrow \frac{p_{2}}{f\left(q_{12}\right)} \geq \frac{p_{1}}{f\left(q_{01}\right)+\delta f\left(q_{12}\right)-\delta f\left(q_{02}\right)} .
\end{aligned}
$$

So $v: g(v)=p_{2} / f\left(q_{12}\right)$ is the lower bound.

Region II,

$$
\frac{f\left(q_{02}\right) p_{1}}{f\left(q_{01}\right)} \leq p_{2} \leq \frac{f\left(q_{12}\right) p_{1}}{f\left(q_{01}\right)+\delta f\left(q_{12}\right)-\delta f\left(q_{02}\right)} .
$$

Find the upper bound of the buy nothing region:

$$
\begin{aligned}
& p_{2} \geq \frac{f\left(q_{02}\right) p_{1}}{f\left(q_{01}\right)} \Leftrightarrow \frac{p_{1}}{f\left(q_{01}\right)} \leq \frac{p_{2}}{f\left(q_{02}\right)}, \text { and } \\
& p_{2} \geq \frac{f\left(q_{02}\right) p_{1}}{f\left(q_{01}\right)} \Rightarrow p_{2} \geq \frac{f\left(q_{12}\right) p_{1}}{f\left(q_{01}\right)} \Leftrightarrow \frac{p_{1}}{f\left(q_{01}\right)} \leq \frac{p_{1}+\delta p_{2}}{f\left(q_{01}\right)+\delta f\left(q_{12}\right)} .
\end{aligned}
$$

So $v: g(v)=p_{1} / f\left(q_{01}\right)$ is the upper bound.

Find the upper bound of the buy in the first-period-only region:

$$
p_{2} \leq \frac{f\left(q_{12}\right) p_{1}}{f\left(q_{01}\right)+\delta f\left(q_{12}\right)-\delta f\left(q_{02}\right)} \Leftrightarrow \frac{\delta p_{2}-p_{1}}{\delta f\left(q_{02}\right)-f\left(q_{01}\right)} \leq \frac{p_{2}}{f\left(q_{12}\right)} .
$$

So $v: g(v)=\left(\delta p_{2}-p_{1}\right) /\left(\delta f\left(q_{02}\right)-f\left(q_{01}\right)\right)$ is the upper bound.

Find the lower bound of the buy in the second-period-only region:

$$
p_{2} \geq \frac{f\left(q_{02}\right) p_{1}}{f\left(q_{01}\right)} \Leftrightarrow \frac{\delta p_{2}-p_{1}}{\delta f\left(q_{02}\right)-f\left(q_{01}\right)} \geq \frac{p_{2}}{f\left(q_{02}\right)} .
$$

So $v: g(v)=\left(\delta p_{2}-p_{1}\right) /\left(\delta f\left(q_{02}\right)-f\left(q_{01}\right)\right)$ is the lower bound.

Find the lower bound of the buy in both periods region:

$$
\begin{aligned}
p_{2} & \leq \frac{f\left(q_{12}\right) p_{1}}{f\left(q_{01}\right)+\delta f\left(q_{12}\right)-\delta f\left(q_{02}\right)} \Rightarrow p_{2} \leq \frac{f\left(q_{02}\right) p_{1}}{f\left(q_{01}\right)+\delta f\left(q_{12}\right)-\delta f\left(q_{02}\right)} \\
& \Leftrightarrow \frac{p_{1}}{f\left(q_{01}\right)+\delta f\left(q_{12}\right)-\delta f\left(q_{02}\right)} \geq \frac{p_{1}+\delta p_{2}}{f\left(q_{01}\right)+\delta f\left(q_{12}\right)}
\end{aligned}
$$

and

$$
p_{2} \leq \frac{f\left(q_{12}\right) p_{1}}{f\left(q_{01}\right)+\delta f\left(q_{12}\right)-\delta f\left(q_{02}\right)} \Leftrightarrow \frac{p_{1}}{f\left(q_{01}\right)+\delta f\left(q_{12}\right)-\delta f\left(q_{02}\right)} \geq \frac{p_{2}}{f\left(q_{12}\right)} .
$$


So $v: g(v)=p_{1} /\left(f\left(q_{01}\right)+\delta f\left(q_{12}\right)-\delta f\left(q_{02}\right)\right)$ is the lower bound.

Region III,

$$
p_{2} \leq \frac{f\left(q_{02}\right) p_{1}}{f\left(q_{01}\right)} .
$$

Find the upper bound of the buy nothing region:

$$
\begin{aligned}
p_{2} & \leq \frac{f\left(q_{02}\right) p_{1}}{f\left(q_{01}\right)} \Leftrightarrow \frac{p_{2}}{f\left(q_{02}\right)} \leq \frac{p_{1}}{f\left(q_{01}\right)}, \text { and } \\
& \quad \frac{f\left(q_{02}\right)}{f\left(q_{01}\right)} \leq \frac{f\left(q_{12}\right)}{f\left(q_{01}\right)+\delta f\left(q_{12}\right)-\delta f\left(q_{02}\right)} \\
p_{2} & \leq \frac{f\left(q_{02}\right) p_{1}}{f\left(q_{01}\right)} \\
\Rightarrow & p_{2} \leq \frac{f\left(q_{12}\right) p_{1}}{f\left(q_{01}\right)+\delta f\left(q_{12}\right)-\delta f\left(q_{02}\right)} \Leftrightarrow \frac{p_{2}}{f\left(q_{02}\right)} \leq \frac{p_{1}+\delta p_{2}}{f\left(q_{01}\right)+\delta f\left(q_{12}\right)} .
\end{aligned}
$$

and

So $v: g(v)=p_{2} / f\left(q_{02}\right)$ is the upper bound.

No consumers buy in the first period only. The upper bound of the first-period-only region is below the lower bound.

$$
p_{2} \leq \frac{f\left(q_{02}\right) p_{1}}{f\left(q_{01}\right)} \Leftrightarrow \frac{p_{1}}{f\left(q_{01}\right)} \geq \frac{\delta p_{2}-p_{1}}{\delta f\left(q_{02}\right)-f\left(q_{01}\right)} .
$$

Find the lower bound of the buy in the second-period-only region:

$$
p_{2} \leq \frac{f\left(q_{02}\right) p_{1}}{f\left(q_{01}\right)} \Leftrightarrow \frac{p_{2}}{f\left(q_{02}\right)} \geq \frac{\delta p_{2}-p_{1}}{\delta f\left(q_{02}\right)-f\left(q_{01}\right)} .
$$

So $v: g(v)=p_{2} / f\left(q_{02}\right)$ is the lower bound.

Find the lower bound of the buy in both periods region:

$$
\begin{aligned}
& p_{2} \leq \frac{f\left(q_{02}\right) p_{1}}{f\left(q_{01}\right)} \Rightarrow p_{2} \leq \frac{f\left(q_{02}\right) p_{1}}{f\left(q_{01}\right)+\delta f\left(q_{12}\right)-\delta f\left(q_{02}\right)} \\
& \Leftrightarrow \frac{p_{1}}{f\left(q_{01}\right)+\delta f\left(q_{12}\right)-\delta f\left(q_{02}\right)} \geq \frac{p_{1}+\delta p_{2}}{f\left(q_{01}\right)+\delta f\left(q_{12}\right)},
\end{aligned}
$$

and

$$
\begin{aligned}
& \left(\frac{f\left(q_{02}\right)}{f\left(q_{01}\right)} \leq \frac{f\left(q_{12}\right)}{f\left(q_{01}\right)+\delta f\left(q_{12}\right)-\delta f\left(q_{02}\right)} \text { and } p_{2} \leq \frac{f\left(q_{02}\right) p_{1}}{f\left(q_{01}\right)}\right) \\
& \Rightarrow \frac{p_{1}}{f\left(q_{01}\right)+\delta f\left(q_{12}\right)-\delta f\left(q_{02}\right)} \geq \frac{p_{2}}{f\left(q_{12}\right)} .
\end{aligned}
$$

So $v: g(v)=p_{1} /\left(f\left(q_{01}\right)+\delta f\left(q_{12}\right)-\delta f\left(q_{02}\right)\right)$ is the lower bound.

Proof of Proposition 2. We start in the second period. We look at the conditions under which the unconstrained optimal solution satisfies the constraints. The optimal solution to the unconstrained second-period problem is $p_{2}=f\left(q_{12}\right) p_{01}^{*} / f\left(q_{01}\right)$. That result follows from the similarity of the objective functions in (4) and (10). From (4), we can see that $0<p_{01}^{*}<f\left(q_{01}\right)$, so Constraints (11) and (12) are met. Constraint (13) is met if

$$
p_{1} \leq \frac{\left(f\left(q_{01}\right)+\delta f\left(q_{12}\right)-\delta f\left(q_{02}\right)\right) p_{01}^{*}}{f\left(q_{01}\right)} .
$$

We will ensure that condition is met by adding it to the first-period problem.

Moving to the first period, by imposing the additional condition (38), we can ignore the effect of second-period revenue in the optimization because the optimal $p_{2}$ is not a function of $p_{1}$. The opti- mal solution to the unconstrained first-period problem is $p_{1}=p_{01}^{*}$. Because the upper bound from (38) is lower than $p_{01}^{*}$ and the firstperiod revenue is concave in $p_{1}$, the optimal solution is to set $p_{1}$ to its upper bound,

$$
p_{1}^{I^{*}}=\frac{\left(f\left(q_{01}\right)+\delta f\left(q_{12}\right)-\delta f\left(q_{02}\right)\right) p_{01}^{*}}{f\left(q_{01}\right)} .
$$

The solution always has sales in both periods because $p_{01}^{*}<$ $f\left(q_{01}\right) \Rightarrow p_{2}^{I^{*}}<f\left(q_{12}\right) \Rightarrow p_{2}^{I^{*}} / f\left(q_{12}\right)<1$, and $\left\{v: g(v) \geq p_{2}^{I^{*}} / f\left(q_{12}\right)\right\}$ buy in both periods.

Proof of Proposition 3. We start in the second period. We look at the conditions under which the unconstrained optimal solution satisfies the constraints. The optimal solution to the unconstrained second-period problem is $p_{2}=f\left(q_{02}\right) p_{01}^{*} / f\left(q_{01}\right)$. That result follows from the similarity of the objective functions in (4) and (23). From (4), we can see that $0<p_{01}^{*}<f\left(q_{01}\right)$, so Constraints (24) and (25) are met. Constraint (26) is met if

$$
p_{1} \geq p_{01}^{*} .
$$

We will ensure that condition is met by adding it to the first-period problem.

Moving to the first period, by imposing the additional condition (39), we can ignore the effect of second-period revenue in the optimization because the optimal $p_{2}$ is not a function of $p_{1}$. The optimal solution to the unconstrained first-period problem is

$$
p_{1}=\frac{\left(f\left(q_{01}\right)+\delta f\left(q_{12}\right)-\delta f\left(q_{02}\right)\right) p_{01}^{*}}{f\left(q_{01}\right)} .
$$

Because the lower bound from (39) is greater than this unconstrained optimal $p_{1}$ and the first-period revenue is concave in $p_{1}$, the optimal solution is to set $p_{1}$ to its lower bound, $p_{1}^{I I *^{*}}=p_{01}^{*}$.

The solution always has sales in both periods because $p_{1}^{I I I^{*}}=p_{01}^{*}$; we imposed the condition

$$
\begin{aligned}
& p_{01}^{*}<f\left(q_{01}\right)+\delta f\left(q_{12}\right)-\delta f\left(q_{02}\right), \\
& \text { and }\left\{v: g(v) \geq \frac{p_{1}^{I I I^{*}}}{f\left(q_{01}\right)+\delta f\left(q_{12}\right)-\delta f\left(q_{02}\right)}\right\}
\end{aligned}
$$

buy in both periods.

\section{References}

Coase, R. H. 1972. Durability and monopoly. J. Law Econom. 15 143-149.

Dhebar, Anirudh. 1994. Durable-goods monopolists, rational consumers, and improving products. Marketing Sci. 13(1) 100-120.

1996. Speeding high-tech producer, meet the balking consumer. Sloan Management Rev. 37(2) 37-49.

Fudenberg, Drew, Jean Tirole. 1998. Upgrades, tradeins, and buybacks. RAND J. Econom. 29(2) 235-258.

$\rightarrow$ Moorthy, K. Sridhar, I. P. L. Png. 1992. Market segmentation, cannibalization, and the timing of product introductions. Management Sci. 38(3) 345-359.

Padmanabhan, V., Surendra Rajiv, Kannan Srinivasan. 1997. New products, upgrades, and new releases: A rationale for sequential product introduction. J. Marketing Res. 34(4) 456-472. 